\title{
Capdepón Verdú, P. y Pastor Comín, J. J. (Eds.) (2015). El patrimonio musical de Castilla-La Mancha: nuevas perspectivas. Madrid: Editorial Alpuerto, 377 pp.
}

En los últimos años, los estudios que revalorizan el patrimonio cultural han vivido un significativo crecimiento gracias a la conciencia ciudadana y a la legislación dirigida a la conservación de los vestigios materiales de carácter plástico-artístico. Sin embargo, si la responsabilidad social y las leyes en torno al citado ámbito son hechos recientes, más modernas aún son las acciones que preservan, investigan y difunden el patrimonio musical. A este respecto, en la actualidad cabe destacar las importantes labores que se están llevando a cabo en la comunidad autónoma de Castilla-La Mancha gracias a la labor que el Centro de Investigación y Documentación Musical (CIDoM-Unidad Asociada al CSIC) de la Universidad de Castilla-La Mancha-Unidad está desempeñando en la actualidad.

Este es el marco en el que debe contextualizarse el volumen El patrimonio musical de Castilla-La Mancha: nuevas perspectivas, editado por los doctores Paulino Capdepón Verdú y Juan José Pastor Comín, profesores de la Universidad de Castilla-La Mancha y codirectores del citado Centro. Se trata del quinto volumen de la colección Investigación y Patrimonio Musical, fruto del convenio firmado en el año 2012 entre el CIDoM-Unidad Asociada al CSIC y la Editorial Alpuerto, tal como se expone en los agradecimientos iniciales del libro que nos atañe. De una extensión de 377 páginas, el volumen cuenta con 10 capítulos, precedido por los agradecimientos y por un prólogo donde se incide en la voluntad de abordar la investigación sobre la riqueza del patrimonio musical de Castilla-La Mancha a través de una mirada caleidoscópica, algo posible debido a la diversidad y a la calidad de testimonios musicales vinculados con la región y al elevado número de profesores del área de Música y Didáctica de la Expresión Musical de la Universidad de Castilla-La Mancha, lo que dio como germen el nacimiento del grupo de investigación Patrimonio Musical de Castilla-La Mancha en el año 2009.

El volumen se inicia con el capítulo de José Luis de la Fuente Charfolé, titulado "El villancico anónimo Al torillo infernal, çagalas (E: Zac b-73/1081) ¿De Juan de Castro y Mallagaray?". El profesor de la Universidad de Castilla-La Mancha expone, de forma detallada y excelentemente documentada, las circunstancias que permitirían la atribución del citado villancico a Juan de Castro y Mallagaray (1570-1632), músico de la provincia de Cuenca, teniendo en cuenta el concepto de autoría en la época y la coincidencia con otras composiciones del músico. Una de las particularidades de este trabajo radica en la convivencia del estudio de las fuentes primarias y de la edición crítica del villancico. Mientras que la primera parte del trabajo ofrece una presentación del manuscrito y un excelente estudio de los datos que permiten llevar a cabo la atribución, atendiendo a otras obras del propio Castro y Mallagaray y teniendo en cuenta los procedimientos 


\section{RESEÑAS DE LIBROS}

compositivos habituales de los siglos XVI y XVII, en la segunda parte se desarrolla la edición crítica de la composición, tan necesaria para posibilitar la ilustración sonora del objeto de estudio y una posible ejecución desde el punto de vista performativo.

El siguiente capítulo, titulado "Villancicos que se han de cantar esta noche: una aproximación a la colección de villancicos de la Catedral de Toledo", también está contextualizado en el ámbito religioso. En este estudio Rafael Javier Moreno Abad, profesor de la Universidad de Castilla-La Mancha, ofrece una mirada de carácter globalizador sobre el ámbito, los rasgos, la funcionalidad, el desarrollo temático y dramático, la evolución y la permanencia en el tiempo del villancico cultivado en torno a la capilla musical del citado templo manchego. Entre otros aspectos señalados en la investigación por R. Javier Moreno Abad, cabe destacar el elevado número de fuentes conservadas, de temática ciertamente diversa, donde se observa una singular convivencia entre el elemento profano y piadoso.

Por su parte, Luis Antonio González Marín, científico titular del Departamento de Ciencias Históricas- Musicología del CSIC, es el autor del capítulo "A vueltas con José de Nebra. Pensamientos sobre autorías y atribuciones". La inserción de este estudio en el presente volumen resulta muy pertinente porque, más allá de aspectos geográficos en torno a su relación con la ciudad de Cuenca -donde vivió cuando su padre se convirtió en organista de la Catedral y donde mantuvo vínculos-, los problemas de atribución de algunas de las obras del citado compositor son extensibles a la comunidad de Castilla-La Mancha, presentando, de este modo, un punto de conexión con el primer capítulo del volumen. Asimismo, Luis Antonio González Marín ofrece un documentado recorrido en torno a las dificultades de atribución de algunas obras de Nebra debido a las coincidencias en los nombres con miembros de su familia y a los testimonios vertidos en el pasado en ediciones modernas, entre otros, con la intención de defender la necesidad de un mayor conocimiento en torno a José de Nebra y al desarrollo de un mayor espíritu crítico como investigadores.

"El patrimonio musical de Talavera de la Reina" es el título de la contribución de Paulino Capdepón Verdú, profesor de la Universidad de Castilla-La Mancha y co-director del Centro de Investigación y Documentación Musical (Unidad Asociada al CSIC). Se trata de una exhaustiva investigación en torno a la actividad musical en la Colegiata de Santa María la Mayor. El capítulo propone un acercamiento a la labor del coro y de la capilla musical polifónica, las dos instituciones principales al respecto, un recorrido por la evolución de la capilla musical de la Colegiata y una presentación de las fuentes musicales albergadas. Además de reivindicar la riqueza patrimonial en la localidad de Talavera de la Reina, un tanto empañada por la actividad de la Catedral Primada de Toledo, la capital de la provincia, el estudio desarrollado por Paulino Capdepón Verdú demuestra de forma magistral la intrínseca relación existente entre la vida musical de la localidad y la desarrollada en el citado templo. 


\section{RESEÑAS DE LIBROS}

El ámbito civil también cuenta con representación a través de diversos capítulos integrados en el presente volumen. Tal es el caso de "El valor documental y musicológico de los fondos de ópera del Museo Nacional del Teatro", de Francisco Manuel López Gómez, profesor de la Universidad Internacional Isabel I. Tras una introducción histórica que explica la existencia de fondos operísticos - pertenecientes en su origen al Teatro Real de Madrid- en la localidad ciudadrealeña de Almagro, es expuesta la actividad musical y empresarial vinculada a la ópera en el Teatro Real y, posteriormente, son presentadas las obras conservadas, tanto de carácter internacional como de producción nacional, siendo éstas últimas más limitadas en número de acuerdo con el gusto del público de la época. Así, este trabajo presenta unas fuentes excepcionales e inéditas de las que, como el propio investigador señala, aquellas obras escritas en castellano son las más desconocidas, de ahí la necesidad de ediciones y de estudios críticos a partir de dichas óperas españolas.

Los músicos de la región de Castilla-La Mancha también cuentan con representación en el volumen que nos ocupa. Así, Octavio J. Peidró Padilla firma el estudio "El compositor castellano-manchego Tomás Barrera Saavedra: 75 aniversario de una música callada". Esta contribución resulta de un gran interés, en primer lugar, por rescatar el nombre de un músico nacido en La Solana (Ciudad Real) que gozó de relevancia nacional en la España de comienzos del siglo XX, especialmente en torno a la música escénica propiamente española, a pesar de que hoy en día no es especialmente recordado. En relación con la visibilidad de Barrera Saavedra (1870-1938) en el contexto cultural y musical de su época, además de aportar aspectos biográficos, Octavio J. Peidró Padilla presenta la trayectoria compositiva del músico como autor de zarzuelas y de género chico y también destaca el papel decisivo de Barrera Saavedra en la creación de la Sociedad de Autores Españoles.

La provincia de Ciudad Real también cuenta con representación en el capítulo titulado "Catalogación del legado histórico musical de Salomón Buitrago (LHMSB) en la Catedral de Ciudad Real”, del profesor Vicente Castellanos Gómez. En primer lugar, este trabajo presenta, de una forma muy pertinente, la figura de Salomón Buitrago (1922-1975) como maestro de capilla y como compositor vinculado al Motu Proprio en Ciudad Real, pero también como un interlocutor relevante en la vida musical de Ciudad Real a través de su labor como profesor de música, como organista, como organizador e impulsor de distintas asociaciones culturales, como director coral y como asesor musical. Sin embargo, el núcleo del trabajo presentado por Castellanos Gómez se centra en exponer, detalladamente, el catálogo del Legado Histórico Musical de Salomón Buitrago, dando cuenta, de este modo, de la riqueza documental y bibliográfica de los citados fondos.

Por su parte, Juan José Pastor Comín, profesor de la Universidad de Castilla-La Mancha y co-director del Centro de Investigación y Documentación Musical (Unidad Asociada al CSIC), es el autor del capítulo "Suba sobre esta máquina el que tuviere ánimo para ello (Don Quijote II, XLI): compositores castellano-manchegos a lomos de Clavileño”. Con este evocador título, sustentado en la visión musical de la novela cervantina a través del personaje de Clavileño - "Clavileño nos ofrece una metáfora de la interpretación 


\section{RESEÑAS DE LIBROS}

musical de la obra cervantina" (p. 265)-, se propone un acercamiento a una selección de composiciones musicales creadas por autores contemporáneos manchegos que han tomado el célebre Don Quijote de la Mancha como fuente de inspiración. Así, Pastor Comín se aproxima a obras de Manuel Ángulo López-Casero (1930), José Antonio Esteban Usano (1968), Manuel Millán de las Heras (1971), Antonio Fernández Reymonde y Julio Sanz Vázquez (1965). Más allá de los aspectos estilísticos de cada autor, y de las particularidades de cada una de las composiciones expuestas, el presente capítulo demuestra de forma magistral cómo la novela de Cervantes continúa siendo una fuente de inspiración en la creación musical contemporánea.

La composición musical de autores manchegos vuelve a ser expuesta en "Ángel Arteaga de la Guía: la recuperación de un gran desconocido”, de Lucía Donoso Madrid. En este capítulo se ofrece una aproximación al citado compositor, nacido en Campo de Criptana (Ciudad Real), que inicialmente se dedicó a la interpretación en el contexto militar y, posteriormente a la composición de música absoluta y también cinematográfica. La investigadora, además de exponer la relevancia del compositor ciudadrealeño a partir de las fuentes documentales a las que ha tenido acceso debido al contacto mantenido con sus herederos, también realiza una revisión de las escasas referencias bibliográficas hacia este compositor de la Generación del 51, concluyendo con la inserción de un catálogo final. En todo caso, el capítulo reivindica la necesidad de un mayor estudio del legado de Ángel Arteaga (1928-1984) debido a su relevancia en el panorama musical español del siglo XX.

El volumen finaliza con el trabajo de Glòria Ballús Casóliva y Antonio Ezquerro Esteban, del Departamento de Ciencias Históricas- Musicología del CSIC. El capítulo, titulado "Los gozos: historia de una composición musical", ofrece una detallada revisión de este género de carácter sacro. En primer lugar, el estudio ofrece una introducción sobre el origen de los Gozos para, posteriormente, mostrar el desarrollo histórico del mismo a lo largo de distintas etapas: desde su aparición hasta la invención de la imprenta, desde la creación del citado invento hasta el Concilio de Trento (1545-1563), desde la segunda mitad del siglo XVI hasta la segunda mitad del siglo XIX en que se producen la creación de la asociación "Amigos de los Gozos" y la aparición del movimiento cecilianista, desde la publicación del Motu Proprio (1903) hasta el Concilio Vaticano II (1962-1965) y desde el citado Concilio hasta la actualidad. Tal como se puede comprobar, y como bien señalan los coordinadores del volumen el prólogo inicial, el trabajo de Ballús y Ezquerro resulta de una gran relevancia por explorar, de una forma tan minuciosa y documentada, en el desarrollo y en los rasgos de género, algo que permitirá otorgar luz de cara a posibles investigaciones sobre los Gozos conservados en los archivos eclesiásticos de Castilla-La Mancha.

Tras lo comentado hasta el momento es evidente que la diversidad temática, expuesta a través de los diferentes capítulos integrados, refleja a la perfección la voluntad de otorgar nuevas perspectivas en torno al patrimonio musical de la región protagonista del volumen. En todo caso, queda claro que El patrimonio musical de Castilla-La Mancha: nuevas perspectivas no solo aporta un mayor conocimiento sobre el rico patrimonio eclesiástico y civil de Castilla-La Mancha sino, además, una pluralidad de enfoques en torno a 


\section{RESEÑAS DE LIBROS}

manifestaciones musicales de épocas diversas que pivotan en torno a la citada región y que son presentadas con rigurosidad metodológica y con un carácter integrador.

Fecha de recepción: 19/10/2016

Fecha de aceptación: 22/11/2016

Virginia Sánchez Rodríguez

Universidad de Castilla-La Mancha 